\title{
SARS-CoV-2 structural features may explain limited neutralizing-antibody responses
}

\author{
Martin F. Bachmann ${ }^{1,2,3 凶}$, Mona O. Mohsen ${ }^{2,3}$, Lisha Zha ${ }^{1}$, Monique Vogel ${ }^{1}$ and Daniel E. Speiser (iD) ${ }^{4 凶}$
}

Neutralizing antibody responses of SARS-CoV-2-infected patients may be low and of short duration. We propose here that coronaviruses employ a structural strategy to avoid strong and enduring antibody responses. Other viruses induce optimal and long-lived neutralizing antibody responses, thanks to 20 or more repetitive, rigid antigenic epitopes, spaced by 5-10 nm, present on the viral surface. Such arrays of repetitive and highly organized structures are recognized by the immune system as pathogenassociated structural patterns (PASPs), which are characteristic for pathogen surfaces. In contrast, coronaviruses are large particles with long spikes (S protein) embedded in a fluid membrane. Therefore, the neutralizing epitopes (which are on the $\mathrm{S}$ protein) are loosely "floating" and widely spaced by an average of about $25 \mathrm{~nm}$. Consequently, recruitment of complement is poor and stimulation of B cells remains suboptimal, offering an explanation for the inefficient and short-lived neutralizing antibody responses.

npj Vaccines (2021)6:2; https://doi.org/10.1038/s41541-020-00264-6

The immune response to severe acute respiratory syndrome coronavirus 2 (SARS-CoV-2) infection is initiated by innate immune activation followed by antigen-specific B- and T-cell responses ${ }^{1}$. An important mechanism protecting from viral disease is the presence of virus-neutralizing antibodies, which is similar for almost all viruses causing acute disease followed by pathogen clearance. In line with this, all currently available anti-viral vaccines are primarily aiming at inducing virus-neutralizing antibodies. Neutralizing antibodies generally block binding of the virus to cellular receptors. In some cases, neutralizing antibodies may prevent conformational changes necessary for fusion of the virus with the cell membrane or proteolytic cleavage. Neutralizing antibodies against SARS-CoV-2 are directed against the spike (S) protein, which contains multiple antigenic epitopes in the receptor-binding domain (RBD) and non-RBD epitopes ${ }^{2}$. A major mechanism of neutralization is to block binding of RBD to angiotensin-converting enzyme 2 (ACE2), the cellular receptor for the virus. The RBD is localized at the tip of the S protein (Fig. 1). The receptor-binding motif (RBM) consists of about 70 aa within the RBD and represents the actual amino acids directly interacting with ACE2.

Neutralizing antibodies are mostly directed against RBD and, in particular, $\mathrm{RBM}^{3-5}$. Indeed, RBD-specific antibodies closely correlate with neutralization in convalescent sera ${ }^{3,6,7}$. Although $S$ is heavily glycosylated, RBD only shows little glycosylation (and one methylation) and the RBM is non-glycosylated, likely facilitating protein-protein interactions with ACE2. This may also indicate that glycosylation of $\mathrm{S}$ is probably not the reason for induction of poor neutralizing antibody responses. $S$ is cleaved by furin and the serine proteases TMPRSS2 and TMPRSS4, enabling fusion of viral and cellular membranes, and consequent entry of viral RNA into the host cell $^{8}$. This cleavage site may also be a target for neutralizing antibodies ${ }^{9,10}$. Overall, the RBD/RBM is the immunological Achilles heel of the virus. Therefore, the virus may have evolved strategies to mitigate induction of neutralizing antibodies against this domain.

\section{DURATION AND QUALITY OF NEUTRALIZING ANTIBODY RESPONSES TO SARS-COV-2}

As for infections with other viruses, COVID-19 patients produce neutralizing antibodies at lower amounts than non-neutralizing ones. There is disagreement about the stability of neutralizing antibodies in COVID-19 patients, with several studies reporting stable persistence ${ }^{11,12}$, whereas others showing that neutralizing antibodies to coronaviruses wane relatively rapidly, or appear late and remain at low titers ${ }^{13-15}$. Some patients may even lack long-lasting antibodies $^{16}$. Indeed, there is increasing evidence that protection from disease can be short-lived: some patients experienced COVID-19 twice within months, proven by a virus-free interval ${ }^{17,18}$.

Antibody titers generally show an early decay after infection, because the first antibody wave is based on short-lived plasma cells $^{19}$. The second wave of antibodies is produced by more durable plasma cells $s^{20}$. Therefore, one cannot directly compare studies that differ in the time points at which antibodies were measured. In addition, studies differ with respect to laboratory methods. The "gold-standard," i.e., neutralization assays that use live virus requires a safety level 3 laboratory, which is not always available. Although useful results are obtained by alternative approaches (pseudotype neutralizing assays or enzyme-linked immunosorbent assays designed to detect RBDspecific antibodies), they are less meaningful than the goldstandard $^{21}$.

The disagreement about the duration of the neutralizing antibody response is not surprising, given the lack of long-term follow up as SARS-CoV-2 has appeared less than one year ago. Nevertheless, patients with minor or no symptoms often have only low and short-lived neutralizing antibody responses ${ }^{13,14}$. In addition, those patients are frequent in the current pandemic.

\footnotetext{
${ }^{1}$ International Immunology Centre, Anhui Agricultural University, Hefei, China. ${ }^{2}$ Department of Rheumatology, Immunology and Allergology, University Hospital Bern, Bern, Switzerland. ${ }^{3}$ Department of BioMedical Research, University of Bern, Bern, Switzerland. ${ }^{4}$ University Hospital and University of Lausanne, Lausanne, Switzerland. ${ }^{\circledR}$ email: martin. bachmann@dbmr.unibe.ch; daniel.speiser@unil.ch
} 


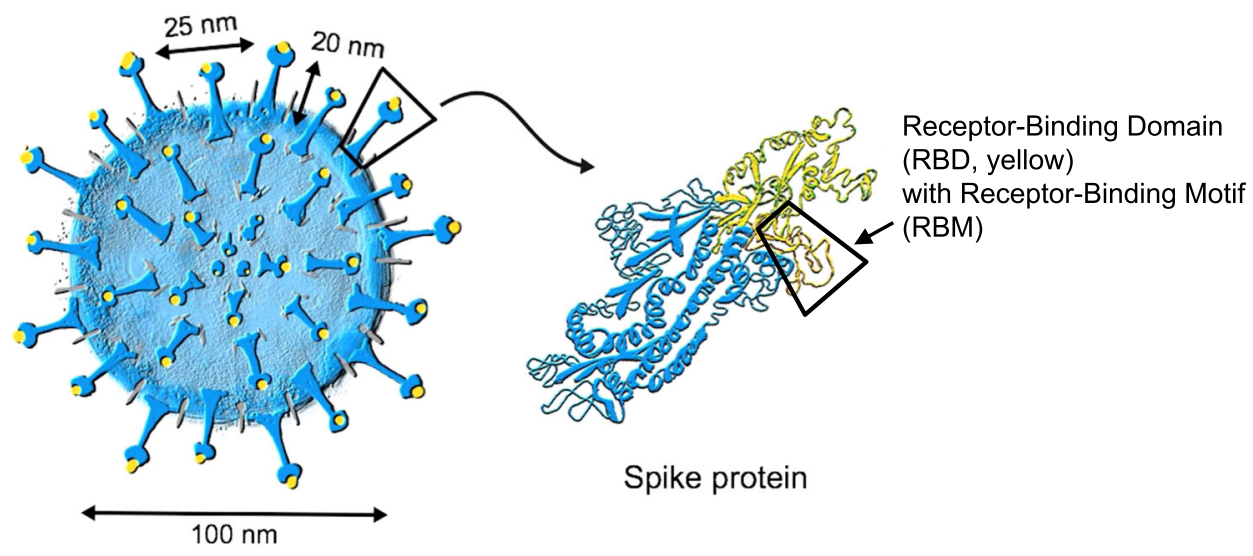

\section{SARS-CoV-2}

Fig. 1 Structure of SARS-CoV-2. Coronaviruses have their names from the typical spikes which are made of the spike (S) protein that is inserted in the lipid bilayer membrane of the virus. The receptor-binding domain (RBD) and its receptor-binding motif (RBM) enable interaction with the cell surface receptor ACE2 mediating entry of the virus into host cells. This can be blocked by neutralizing antibodies. Therefore, most neutralizing epitopes are located on RBD/RBM. Besides the S protein, SARS-CoV-2 has two further viral surface proteins (not shown): envelope (E) and matrix (M).

Milder symptoms are observed when viral replication is restricted to the upper respiratory track. This occurs also in the usually mild common cold infections caused by the endemic seasonal coronaviruses, typically during winter and spring time ${ }^{20}$. For these viruses, antibody responses that protect from disease are shortlived ${ }^{14}$, in the range of 1 year $^{22}$ or less ${ }^{23}$, and infections occur regularly, including re-infections with the same virus ${ }^{15,22,24}$.

Until last year, there existed only two coronaviruses that frequently cause severe disease, SARS-CoV-1 and Middle East Respiratory Syndrome Coronavirus (MERS-CoV). Neutralizing antibody responses to SARS-CoV-1 can be measured in most patients ${ }^{25,26}$ but may gradually disappear after recovery ${ }^{27}$. More is known about the MERS-CoV. This virus keeps on circulating in its natural hosts, the dromedary populations, and animals may experience re-infections. The camels have a high seroprevalence $(>90 \%)$, but virus transmission is not blocked by previous infection $^{28}$.

Different other reasons may hamper the immune response to coronaviruses, e.g., those concerning the innate immune system, which is key for early activation of inflammatory cells and cytokines. SARS-CoV-2 may inhibit dendritic cells ${ }^{29}$ and interferon (IFN)-I/III responses ${ }^{30,31}$. Relatively small percentages of patients with severe COVID-19 bear various genetic variants that compromise innate immune mechanisms ${ }^{32}$, in particular, type I IFN pathways ${ }^{33}$, or have autoantibodies against type I IFNs ${ }^{34}$, which are likely aggravating diseases severity. Regarding $T$ cells, several studies showed impaired T-cell responses including CD4 helper and regulatory $T$ cells ${ }^{29,35}$. This point could be important, as T cells may contribute to protection from disease, although this is not proven $^{36}$. It is also necessary to state that for neutralizing antibodies, there is currently no proof that they indeed mediate protection from COVID-19. For most current vaccines, neutralizing antibodies are considered as correlate of protection from disease, although they do not necessarily equate to the only mechanism of protection. Finally, non-neutralizing antibodies such as those that fix complement on the viral surface or mediate antibodydependent cellular cytotoxicity may also play a role, although this is not yet clear ${ }^{36}$.

In the following, we propose that structural adaption of the virus family is co-responsible for the inefficiency of neutralizing antibody responses to the $S$ protein of SARS-CoV-2, in particular RBD/RBM.

\section{STRUCTURE FUNCTION CONSIDERATIONS FOR SARS-COV-2}

Most viruses have highly organized, repetitive and rigid surfaces ${ }^{37}$. Typical RNA viruses cannot build up complex surfaces because of their limited genome of around 10,000 nucleotides (10 kb). Their capsid usually consists of multiple copies of only one or two proteins, often arranged in icosahedral symmetry ${ }^{38}$, readily and efficiently inducing neutralizing antibody responses ${ }^{39}$. As the vertebrate body is by and large devoid of such extracellular repetitive and organized structures, the immune system has evolved to recognize such antigen organization as a pathogenassociated structural pattern (PASP) ${ }^{40}$. In the 1970s, it was found that optimal immune responses are induced by at least 12-16 epitopes spaced by $5-10 \mathrm{~nm}$, called the immunon ${ }^{41}$. Figure $2 \mathrm{~A}$ shows a typical RNA virus, with a diameter of $30 \mathrm{~nm}$ and 180 copies of a single coat protein spaced by about $5 \mathrm{~nm}$. Such viral particles efficiently cross-link B-cell receptors ${ }^{42,43}$ and are recognized by natural IgM, which induces the classical pathway of complement activation. This facilitates binding of viral particles to complement receptors followed by B-cell-mediated deposition on follicular dendritic cells causing efficient germinal center formation $^{44}$. Furthermore, complement-dependent stimulation of CD21 on B cells facilitates induction of long-lived plasma cells, which is essential for durable antibody responses ${ }^{45}$. There is a vast literature confirming these considerations for human vaccines, where repetitiveness is important for inducing long-lived antibody responses 37,46 . Hence, repetitive, rigid structures spaced by 5-10 nm are optimal for complement and B-cell activation, resulting in durable antibody responses.

Figure $2 \mathrm{~A}$ outlines the structure of SARS-CoV-2. It is immediately evident that the structure of this coronavirus is quite different. The virion has a relatively large body with a diameter of $100 \mathrm{~nm}$ (rather than 30-50 nm). Importantly, the S protein, which has a length of about $20 \mathrm{~nm}$, is present rather scarcely, floating in a sea of lipid bilayer. As mentioned above, RBD is sitting at the top of S; therefore, some $70 \mathrm{~nm}$ away from the center. The viral surface area, in which RBD is moving within a two-dimensional space, $20 \mathrm{~nm}$ away from the lipid bilayer, can be calculated as $4 \times \pi r^{2}=$ $4 \times \pi 70 \mathrm{~nm}^{2}=$ ca $62,000 \mathrm{~nm}^{2}$. Assuming an average number of ca. $100 \mathrm{~S}$ per virion, each $\mathrm{S}$ covers a surface area of about $620 \mathrm{~nm}^{2}$. This leads to a grid-length of $25 \mathrm{~nm}$, which indicates that $S$ is spaced by an average of $25 \mathrm{~nm}$ (Fig. 2C) rather than the $5-10 \mathrm{~nm}$ needed for optimal B-cell responses. Epitopes spaced by this large distance in a non-rigid manner are inefficient in cross-linking $\mathrm{B}$-cell receptors or recruiting natural IgM antibodies, required for 


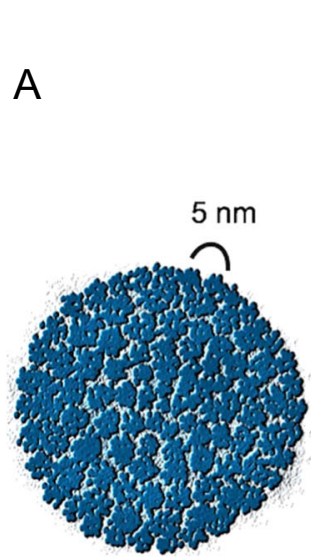

RNA-Virus
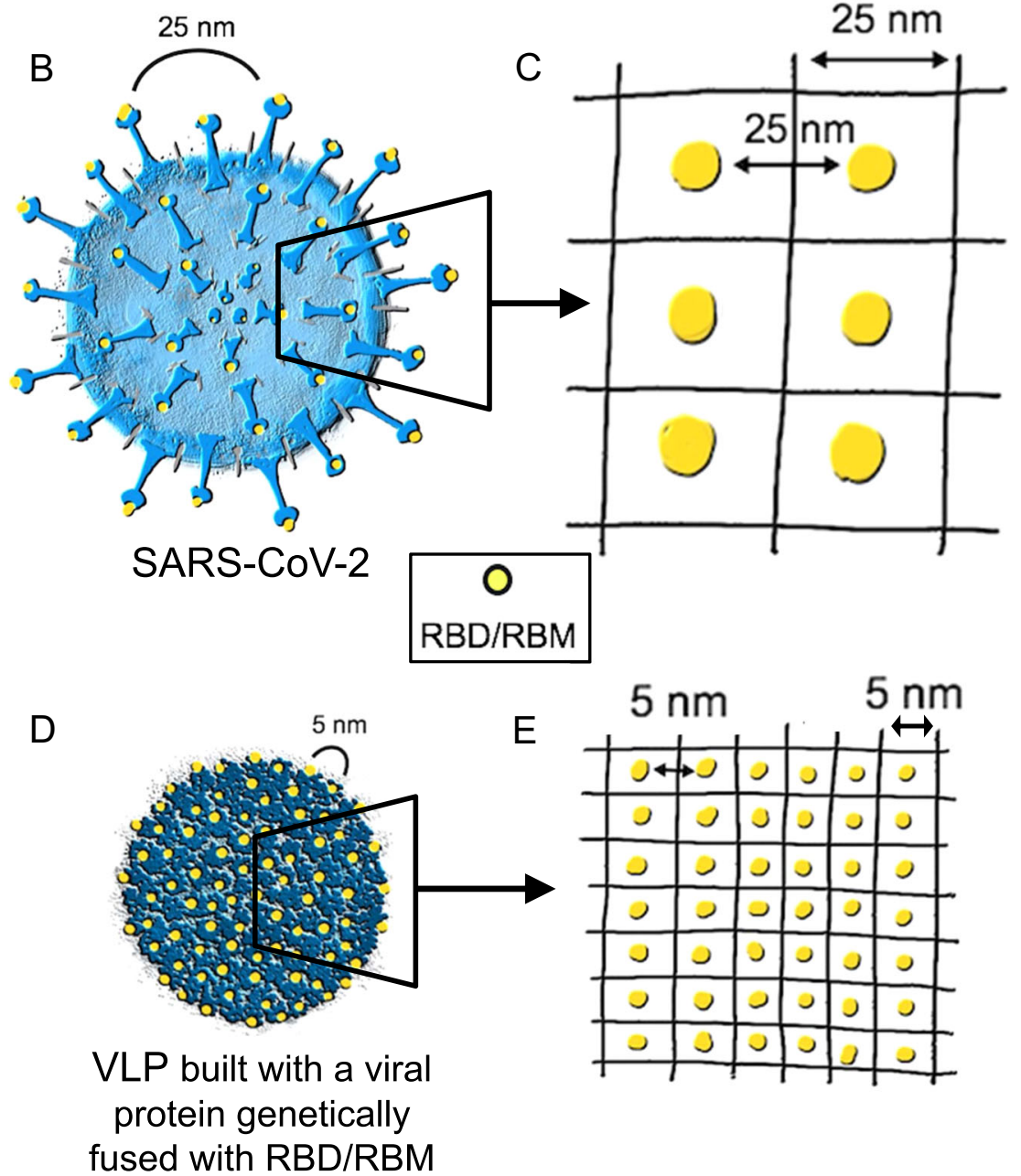

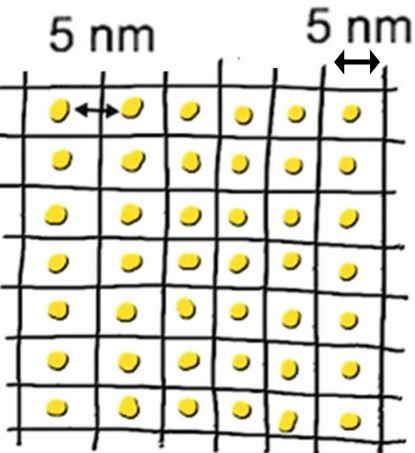

fused with RBD/RBM

Fig. 2 Distances between neutralizing epitopes. A An example of a classical RNA virus with a capsule made of multiple copies of only one protein that are rigidly structured, displaying highly immunogenic repetitive neutralizing epitopes spaced by $5-10 \mathrm{~nm}$. This virus is built with 180 monomers and has a total viral diameter of $30 \mathrm{~nm}$. B, C A coronavirus with its $S$ proteins, showing the distance of the neutralizing epitopes of about $25 \mathrm{~nm}$, which is large and unfavorable for triggering antibody responses. D, E A virus-like particle (VLP) built by a viral protein into which the RBM of SARS-CoV-2 is genetically inserted. This VLP displays the neutralizing epitopes with an optimized spacing of $5 \mathrm{~nm}$.

complement activation and induction of long-live plasma cells. Hence, SARS-CoV-2 dilutes it's Achilles heel, RBD on S, in a sea of lipids and other proteins, avoiding potent neutralizing antibody responses.

The $\mathrm{S}$ protein forms a trimer. Consequently, the RBD will display three identical epitopes favorably spaced by about $3-5 \mathrm{~nm}$. As discussed above, three epitopes are, however, not enough to optimally activate B cells. On the contrary, epitopes occurring in low numbers inhibit, rather than activate, B-cell responses. Indeed, Dintzis et al. ${ }^{47}$ concluded that increasing epitope density in a molecular structure increases its immunogenicity if the threshold number of $\sim 20$ is reached. In contrast, increasing the density in a molecular structure below the threshold number increases its tolerogenicity ${ }^{47}$. Thus, trimeric RBD may reduce rather than increase neutralizing antibody responses.

An additional consideration is the length of the viral genome, as SARS-CoV-2 is encoded by 30,000 RNA nucleotides rather than the usually about 10,000 nucleotides seen for most other RNA viruses. Indeed, the longer genome of coronaviruses includes an RNA proofreading system, required for keeping the viral population viable based on sufficient genome stability ${ }^{48}$. Hence, in contrast to other RNA viruses, coronaviruses can build up relatively complex surfaces that allow evasion from immune recognition as a PASP and induction of enduring neutralizing antibody responses.

Besides the non-enveloped viruses, also many enveloped viruses (Influenza, Rabies, Sindbis, and Vesicular Stomatitis Virus) display highly immunogenic antigen arrays. Lytic viruses generally induce potent antibody responses and produce serotypes $^{37}$. A notable exception were adenoviruses, which, similar to SARS-CoV-2, are lytic but also do not form serotypes. Parallel to coronaviruses, adenoviruses also dilute out the neutralizing epitopes on the surface of the virion thereby apparently avoiding stringent long-term neutralization (hence, no serotype formation). Similar to coronaviruses, adenoviruses have a proofreading replication system, as they are DNA viruses ${ }^{49}$. Hence, the presence of proofreading may allow viruses to escape immune recognition as a PASP.

We have previously discussed ${ }^{37}$ that viral structure predicts host antibody responses and serotype formation. An interesting observation was that viruses with highly organized and rigid surfaces induce T-cell independent antibody responses and form serotypes, whereas viruses with a less rigid structure avoid potent antibody responses and do not form serotypes. However, the "dilution" of the neutralizing epitopes performed by coronaviruses, 
as described in this study, is a unique strategy in the world of RNA viruses.

\section{IMPLICATIONS FOR VACCINE DESIGN}

If the inefficient and short-lived neutralizing antibody responses induced by SARS-CoV-2 are indeed caused by the unusually large distance between neutralizing epitopes embedded in a fluid membrane, this has important implications for vaccine design. Specifically, by simple genetic extraction of RBD or RBM from SARS-CoV-2 followed by grafting onto highly repetitive and immunogenic nanoparticles or virus-like particles (VLPs), one may render the poorly immunogenic RBD/RBM into a highly immunogenic version of it (Fig. 2D), with high numbers of accessible epitopes at optimal distancing (Fig. 2E). Indeed, chemical coupling or conjugation by the Spy-Catcher or similar methods of RBD to VLPs results in highly immunogenic vaccine candidates that stimulate production of high levels of neutralizing antibodies in test animals ${ }^{50,51}$. An alternative strategy, which facilitates large scale production, is represented by genetic fusion of RBD/RBM onto VLP-surfaces. Such approaches may represent attractive options that we and others are currently following ${ }^{50,52,53}$.

\section{CONCLUSION}

SARS-CoV-2 induces inefficient neutralizing antibody responses that are short-lived. In contrast to the other RNA virus families, which display arrays of neutralizing epitopes spaced by $5-10 \mathrm{~nm}$ in a rigid manner, SARS-CoV-2 displays a low number of neutralizing epitopes spaced by $25 \mathrm{~nm}$ in a non-rigid manner, as the $\mathrm{S}$ protein is embedded in a fluid membrane. Hence, SARSCoV-2 escapes an efficient neutralizing antibody response by structurally avoiding immunogenic display of its neutralizing epitopes.

Received: 16 October 2020; Accepted: 23 November 2020; Published online: 04 January 2021

\section{REFERENCES}

1. Thevarajan, I. et al. Breadth of concomitant immune responses prior to patient recovery: a case report of non-severe COVID-19. Nat. Med. 26, 453-455 (2020).

2. Brouwer, P. J. M. et al. Potent neutralizing antibodies from COVID-19 patients define multiple targets of vulnerability. Science, https://doi.org/10.1126/science. abc5902 (2020).

3. Berry, J. D. et al. Neutralizing epitopes of the SARS-CoV S-protein cluster independent of repertoire, antigen structure or mAb technology. MAbs 2, 53-66 (2010).

4. Shi, R. et al. A human neutralizing antibody targets the receptor-binding site of SARS-CoV-2. Nature 584, 120-124 (2020).

5. Ju, B. et al. Human neutralizing antibodies elicited by SARS-CoV-2 infection. Nature 584, 115-119 (2020).

6. Barnes, C. O. et al. Structures of human antibodies bound to SARS-CoV-2 spike reveal common epitopes and recurrent features of antibodies. Cell 182, 828-842. e16 (2020).

7. Brigger, D. et al. Accuracy of serological testing for SARS-CoV-2 antibodies: first results of a large mixed-method evaluation study. Allergy, https://doi.org/ 10.1111/all.14608 (2020)

8. Zang, R. et al. TMPRSS2 and TMPRSS4 promote SARS-CoV-2 infection of human small intestinal enterocytes. Sci. Immunol. 5 (2020).

9. Sun, Z. et al. Mass spectrometry analysis of newly emerging coronavirus HCoV-19 spike protein and human ACE2 reveals camouflaging glycans and unique posttranslational modifications. Engineering. https://doi.org/10.1016/j.eng.2020.07.014 (2020).

10. Grant, O. C., Montgomery, D., Ito, K. \& Woods, R. J. Analysis of the SARS-CoV-2 spike protein glycan shield: implications for immune recognition. Sci. Rep. 10, 14991 (2020).
11. Gudbjartsson, D. F. et al. Humoral immune response to SARS-CoV-2 in Iceland. $N$. Engl. J. Med. https://doi.org/10.1056/NEJMoa2026116 (2020).

12. Isho, B. et al. Persistence of serum and saliva antibody responses to SARS-CoV-2 spike antigens in COVID-19 patients. Sci. Immunol. 5, eabe5511 (2020).

13. Tay, M. Z., Poh, C. M., Rénia, L., Macary, P. A. \& Ng, L. F. P. The trinity of COVID-19: immunity, inflammation and intervention. Nat. Rev. Immunol. 20, 363-374 (2020).

14. Sariol, A. \& Perlman, S. Lessons for COVID-19 immunity from other coronavirus infections. Immunity 53, 248-263 (2020).

15. Jeyanathan, M. et al. Immunological considerations for COVID-19 vaccine strategies. Nat. Rev. Immunol. 20, 615-632 (2020).

16. CGTN. Recovered coronavirus patients are still prone to reinfection. Available at: https://www.youtube.com/watch?v=GZ99J7mlalQ. Accessed 10 Aug 2020.

17. Bentivegna, E. et al. New IgM seroconversion and positive RT-PCR test after exposure to the virus in recovered COVID-19 patient. J. Med. Virol. https://doi.org/ 10.1002/jmv.26160 (2020).

18. Ledford, H. COVID-19 reinfection: three questions scientists are asking. Nature 585, 168-169 (2020).

19. Alter, G. \& Seder, R. The power of antibody-based surveillance. N. Engl. J. Med. https://doi.org/10.1056/NEJMe2028079 (2020).

20. Amanna, I. J., Carlson, N. E. \& Slifka, M. K. Duration of humoral immunity to common viral and vaccine antigens. N. Engl. J. Med. 357, 1903-1915 (2007).

21. Seow, J. et al. Longitudinal observation and decline of neutralizing antibody responses in the three months following SARS-CoV-2 infection in humans. Nat. Microbiol. 5, 1598-1607 (2020).

22. Reed, S. E. The behaviour of recent isolates of human respiratory coronavirus in vitro and in volunteers: evidence of heterogeneity among $229 \mathrm{E}-$-related strains. J. Med. Virol. 13, 179-192 (1984).

23. Callow, K. A., Parry, H. F., Sergeant, M. \& Tyrrell, D. A. The time course of the immune response to experimental coronavirus infection of man. Epidemiol. Infect. 105, 435-446 (1990).

24. Galanti, M. \& Shaman, J. Direct observation of repeated infections with endemic coronaviruses. J. Infect. Dis. https://doi.org/10.1093/infdis/jiaa392 (2020).

25. Temperton, N. J. et al. Longitudinally profiling neutralizing antibody response to SARS coronavirus with pseudotypes. Emerg. Infect. Dis. 11, 411-416 (2005).

26. Nie, Y. et al. Neutralizing antibodies in patients with severe acute respiratory syndrome-associated coronavirus infection. J. Infect. Dis. 190, 1119-1126 (2004).

27. Cao, W.-C., Liu, W., Zhang, P.-H., Zhang, F. \& Richardus, J. H. Disappearance of antibodies to SARS-associated coronavirus after recovery. N. Engl. J. Med. 357, 1162-1163 (2007).

28. Hemida, M. G. et al. Longitudinal study of Middle East Respiratory Syndrome coronavirus infection in dromedary camel herds in Saudi Arabia, 2014-2015. Emerg. Microbes Infect. 6, e56 (2017).

29. Zhou, R. et al. Acute SARS-CoV-2 infection impairs dendritic cell and T cell responses. Immunity 53, 864-877.e5 (2020).

30. Blanco-Melo, D. et al. Imbalanced host response to SARS-CoV-2 drives development of COVID-19. Cell 181, 1036-1045.e9 (2020).

31. Sa Ribero, M., Jouvenet, N., Dreux, M. \& Nisole, S. Interplay between SARS-CoV-2 and the type I interferon response. PLoS Pathog. 16, e1008737 (2020).

32. Beck, D. B. \& Aksentijevich, I. Susceptibility to severe COVID-19. Science 370, 404-405 (2020).

33. Zhang, Q. et al. Inborn errors of type I IFN immunity in patients with lifethreatening COVID-19. Science 370, eabd4570 (2020).

34. Bastard, P. et al. Auto-antibodies against type I IFNs in patients with lifethreatening COVID-19. Science, https://doi.org/10.1126/science.abd4585 (2020).

35. Meckiff, B. J. et al. Imbalance of regulatory and cytotoxic SARS-CoV-2-reactive CD4(+) T cells in COVID-19. Cell, https://doi.org/10.1016/j.cell.2020.10.001 (2020).

36. Peiris, M. \& Leung, G. M. What can we expect from first-generation COVID-19 vaccines? Lancet, https://doi.org/10.1016/S0140-6736(20)31976-0 (2020).

37. Bachmann, M. F. \& Zinkernagel, R. M. The influence of virus structure on antibody responses and virus serotype formation. Immunol. Today 17, 553-558 (1996).

38. Mohsen, M. O., Augusto, G. \& Bachmann, M. F. The 3Ds in virus-like particle based-vaccines: 'Design, Delivery and Dynamics'. Immunol. Rev. 296, 155-168 (2020).

39. Bachmann, M. F. \& Zinkernagel, R. M. Neutralizing antiviral B cell responses. Annu. Rev. Immunol. 15, 235-270 (1997).

40. Bachmann, M. F. \& Jennings, G. T. Vaccine delivery: a matter of size, geometry, kinetics and molecular patterns. Nat. Rev. Immunol. 10, 787-796 (2010).

41. Dintzis, H. M., Dintzis, R. Z. \& Vogelstein, B. Molecular determinants of immunogenicity: the immunon model of immune response. Proc. Natl Acad. Sci. USA 73, 3671-3675 (1976).

42. Bachmann, M. F. et al. The influence of antigen organization on B cell responsiveness. Science 262, 1448-1451 (1993).

43. Chackerian, B., Lowy, D. R. \& Schiller, J. T. Conjugation of a self-antigen to papillomavirus-like particles allows for efficient induction of protective autoantibodies. J. Clin. Invest. 108, 415-423 (2001). 
44. Link, A. et al. Innate immunity mediates follicular transport of particulate but not soluble protein antigen. J. Immunol. 188, 3724-3733 (2012).

45. Gatto, D. et al. Complement receptors regulate differentiation of bone marrow plasma cell precursors expressing transcription factors Blimp-1 and XBP-1. J. Exp. Med. 201, 993-1005 (2005).

46. Slifka, M. K. \& Amanna, I. J. Role of multivalency and antigenic threshold in generating protective antibody responses. Front. Immunol. 10, 956 (2019).

47. Dintzis, R. Z., Middleton, M. H. \& Dintzis, H. M. Studies on the immunogenicity and tolerogenicity of T-independent antigens. J. Immunol. 131, 2196-2203 (1983).

48. Sevajol, M., Subissi, L., Decroly, E., Canard, B. \& Imbert, I. Insights into RNA synthesis, capping, and proofreading mechanisms of SARS-coronavirus. Virus Res. 194, 90-99 (2014).

49. Wang, D. \& Hawley, D. K. Identification of a $3^{\prime}->5^{\prime}$ exonuclease activity associated with human RNA polymerase II. Proc. Natl Acad. Sci. USA 90, 843-847 (1993).

50. Zha, L. et al. Development of a COVID-19 vaccine based on the receptor binding domain displayed on virus-like particles. Preprint at https://doi.org/10.1101/ 2020.05.06.079830 (2020).

51. Fougeroux, C. et al. Capsid-like particles decorated with the SARS2-CoV-2 receptor-binding domain elicit strong virus neutralization activity. Res. Gate 1-23, https://doi.org/10.21203/rs.3.rs-45062/v1 (2020).

52. Walls, A. C. et al. Elicitation of potent neutralizing antibody responses by designed protein nanoparticle vaccines for SARS-CoV-2. Cell 183, 1367-1382.e17 (2020).

53. Keech, $C$. et al. Phase 1-2 trial of a SARS-CoV-2 recombinant spike protein nanoparticle vaccine. N. Engl. J. Med. https://doi.org/10.1056/NEJMoa2026920 (2020).

\section{ACKNOWLEDGEMENTS}

We thank Kaspars Tars for help with calculating viral dimensions and all members of the Bachmann lab for their numerous contributions. Worldwide, many scientists have made seminal contributions to this field, which we could not cite due to space limitations. This work was supported by the Swiss National Science Foundation (SNF grants 31003A-149925 and 310030-179459), the Universities of Lausanne and Bern, Switzerland, and the International Immunology Centre, Anhui Agricultural University, Hefei, China.

\section{AUTHOR CONTRIBUTIONS}

M.F.B. designed the manuscript. M.F.B. and D.E.S. wrote the main parts. M.F.B., M.O.M., and D.E.S. generated the figures. M.F.B., M.O.M., L.Z., M.V., and D.E.S. did literature research, editing, and revisions.

\section{COMPETING INTERESTS}

M.F.B. and M.O.M. own shares and/or receive salaries of Saiba $\mathrm{GmbH}$, which is involved in the development of a vaccine against COVID-19. L.Z., M.V., and D.E.S. declare no competing interests.

\section{ADDITIONAL INFORMATION}

Correspondence and requests for materials should be addressed to M.F.B. or D.E.S.

Reprints and permission information is available at http://www.nature.com/ reprints

Publisher's note Springer Nature remains neutral with regard to jurisdictional claims in published maps and institutional affiliations.

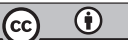

Open Access This article is licensed under a Creative Commons Attribution 4.0 International License, which permits use, sharing adaptation, distribution and reproduction in any medium or format, as long as you give appropriate credit to the original author(s) and the source, provide a link to the Creative Commons license, and indicate if changes were made. The images or other third party material in this article are included in the article's Creative Commons license, unless indicated otherwise in a credit line to the material. If material is not included in the article's Creative Commons license and your intended use is not permitted by statutory regulation or exceeds the permitted use, you will need to obtain permission directly from the copyright holder. To view a copy of this license, visit http://creativecommons. org/licenses/by/4.0/.

(c) The Author(s) 2021 